\title{
FORUM
}

\section{Sicherheit, Macht und die transatlantischen Beziehungen}

\author{
Jackson Janes*
}

Wie wird globale Sicherheit im 21. Jahrhundert definiert und was kann die Europäische Union zum Erhalt von Sicherheit beitragen?

Das Problem bei der Definition von globaler Sicherheit ist die Tatsache, dass wir in einer Welt der Ungleichgewichte und Asymmetrien leben. Die Kräfte der Globalisierung schnüren Länder und Bevölkerungen enger aneinander, erzeugen jedoch zugleich Zentrifugalkräfte in und unter den Nationen, während diese sich anzupassen versuchen. In den vergangenen zehn Jahren haben wir auf dem Balkan, in Afrika und Asien zahlreiche schmerzvolle Beispiele der dunklen Seite einer sich globalisierenden Welt gesehen, die von extremer Armut bis hin zu ethnischen Spannungen reichen. Andere Regionen wiederum, allen voran die Europäische Union, stellen eine Erfolgsgeschichte der Globalisierung dar.

\section{Machtkonstellationen und Sicherheitsparameter im globalen Wandel}

Aber sehen wir uns das globale Bild an: In den kommenden zwei Jahrzehnten werden China und Indien als Großmächte auf den Plan treten. Mit ihrem wirtschaftlichen Wachstum, ihrer militärischen Stärke und ihrer enormen Bevölkerung mit ausgebildeten Arbeitskräften werden beide Länder die Weltwirtschaft mitprägen. Andere Regionen wie Südamerika können ebenfalls mächtige Spieler, wie zum Beispiel Brasilien, auf der Weltbühne hervorbringen. Dagegen werden möglicherweise Nationen in Afrika oder in Teilen des Nahen und Mittleren Ostens in den Sog ethnischen oder religiösen Unfriedens geraten oder durch Krankheiten und Armut noch weiter zurückfallen. Im 20. Jahrhundert kämpften wir gegen Staaten, die ein zu großes Drohpotenzial aufwiesen. In diesem Jahrhundert sehen wir uns Staaten gegenüber, die zu schwach sind - von uns als ,failing states` bezeichnet -, und die sichere Häfen für diejenigen bieten, die diese Schwäche auszunutzen suchen.

Konflikte um Energievorräte, nationalistische Spannungen und separatistische Bewegungen werden sich fortsetzen und dem Terrorismus als Nährboden dienen. Sie werden die Verwundbarkeit erhöhen, damit aber auch den Ruf nach Schutz und Sicherheitsmaßnahmen zwischen und innerhalb von Staaten und Regionen. Der Kampf um sichere Energiequellen wird ein Hauptproblem für alle führenden Staaten sein und sich auf die Außenpolitik eines jeden einzelnen auswirken.

Alle diese Entwicklungen werden ein Umdenken unserer Definition von Sicherheit verlangen. So wie wir nicht länger vom Ost-West-Konflikt in Europa sprechen, dessen Trennlinie einst mitten durch Berlin verlief, so obsolet erscheint unsere von Konzepten aus der Zeit des Kalten Krieges, wie dem der Dritten Welt, des Nord-Süd-Konflikts und anderer Konstellationen, beeinflusste Betrachtungsweise. Konzepte wie nationale Souveränität befinden sich auf Grund der zunehmenden Verflechtungen im Zuge der Globalisierung ebenfalls im Wan-

* Dr. Jackson Janes, Executive Director, American Institute for Contemporary German Studies, Johns Hopkins University, Washington D.C. Der Beitrag basiert auf einem Vortrag des Autors (Originaltext Englisch) im Rahmen der vom IEP durchgeführten Konferenz, Young Faces“ am 20. Januar 2005 in Berlin. Die Konferenz fand zur Eröffnung des gemeinsamen Programms „European Foreign and Security Policy Studies“ der VolkswagenStiftung, der Compagnia di San Paolo, Turin/Italien, und des Riksbankens Jubileumsfond, Stockholm/ Schweden statt. 
del, manchmal aus freien Stücken und manchmal erzwungen durch wirtschaftliche und technische Entwicklungen, die unsere Wahlmöglichkeiten neu prägen, sowie durch neue Formen der Verwundbarkeit. Nichtstaatliche Akteure wie globale Unternehmen und Nichtregierungsorganisationen oder al-Qaida und das organisierte Verbrechen gewinnen immer mehr an Bedeutung, im Guten und allzu oft im Schlechten. ${ }^{1}$

Die Mittel, mit denen wir unsere Außenpolitik gestalten, einschließlich der nach dem Zweiten Weltkrieg geschaffenen internationalen Institutionen, wie zum Beispiel der UNO, der Weltbank und des IWF, werden sich an die Herausforderungen der neuen Sicherheitsparameter anpassen müssen. Das erfordert enorme Investitionen in die wirtschaftliche, politische und soziale Entwicklung jener Weltregionen, die als Brutstätte von Hass und Terrorismus gelten. Das 21. Jahrhundert wird das Aufgebot der politischen Akteure auf der Weltbühne neu arrangieren.

Wie kann der ,Westen' in diesem Zukunftsszenario begriffen werden und welche Rolle spielen die transatlantischen Beziehungen?

\section{Transatlantische Differenzen in der Wahrnehmung und Lösung von Konflikten}

Vorauszuschicken ist, dass in absehbarer Zukunft die Vereinigten Staaten auf Grund ihrer Vorherrschaft in so vielen Bereichen der mächtigste Spieler bleiben werden. Doch die amerikanische Vorherrschaft gerät von vielen Seiten zunehmend unter Druck. ${ }^{2}$

Mit einer anfällig auf die Schwankungen einer globalisierten Welt reagierenden Wirtschaft, einer hochgradigen Abhängigkeit von ausländischem Öl und angesichts der Herausforderungen in Bereichen der Umweltpolitik, des Außenhandels und der sozialen Belange werden die USA bei der Verfolgung ihrer Interessen sicherstellen müssen, dass sie Konflikte bewältigen, Kooperation fördern und mit Bedrohungen in einem komplizierten Netz gegenseitiger Abhängigkeiten umgehen können. Darin liegt eine Herausforderung sowohl für die Außenpolitik der USA, als auch für die innenpolitische Debatte über diesbezügliche Prioritäten.

Derzeit stellen die transatlantischen Verbindungen die stärksten Beziehungen dar, die die USA in diesem Netz von Interdependenzen unterhalten. Sie machen mehr als die Hälfte des weltweiten Handels und Investitionen in Höhe von 2,5 Billionen Dollar aus. Angesichts von beinahe 800 Millionen Verbrauchern zu beiden Seiten des Atlantiks steht bei der Bewahrung stabiler transatlantischer Beziehungen buchstäblich eine Menge auf dem Spiel, was aber unvermeidliche Konflikte und Wettbewerb innerhalb dieses verflochtenen Netzes nicht ausschließt. $^{3}$

Doch aus der gegenseitigen wirtschaftlichen Abhängigkeit muss nicht unmittelbar die Einsicht in eine gegenseitige politische Abhängigkeit erwachsen. Dies ist teilweise darauf zurückzuführen, dass es zunehmend schwieriger wird, in Sicherheitsfragen, mit denen wir heute konfrontiert werden, Innen- und Außenpolitik voneinander zu trennen. Unterscheidet sich die jeweilige Wahrnehmung von Sicherheitsproblemen in der entsprechenden innenpolitischen Debatte auf beiden Seiten des Atlantiks, dann gibt es reichlich Raum für Friktionen.

1 Vgl. Susan Stern/Elisabeth Seligmann (Hrsg.): The Partnership Principle - New Forms of Governance in the 21 st Century, Alfred Herrhausen Society for International Dialogue, London 2004 und 21st Sinclair House Debate: Beyond the State? 'Foreign Policy' by Companies and NGOs, Herbert-Quandt-Stiftung, August 2004.

2 Vgl. Niall Ferguson: Colossus - The Price of America's Empire, New York 2004; und Thomas P. M. Barnett: The Pentagon's New Map - War and Peace in the Twenty-First Century, New York 2004.

3 Vgl. Dan Hamilton/Joseph Quinlan: Partners in Prosperity: The Changing Geography of the Transatlantic Economy, Center for Transatlantic Relations, Johns Hopkins University - SAIS, Washington D.C. 2004. 
Dergleiche Friktionen zeigten sich deutlich nach dem 11. September, als die USA rasch die Auffassung vertraten, sie befänden sich im Kriegszustand, während die Europäer die terroristische Bedrohung zwar insgesamt als Gefahr ansahen, aber nicht als ein Subjekt, dem man den Krieg erklären könnte, sondern eher als ein Mittel, das Gruppen gewählt hatten, um ihre Ziele zu erreichen. Während man sich über die Gefahren weitgehend einig war, gab es keinen Konsens, wie man diesen Gefahren begegnen sollte.

Was die Bush-Administration unter Sicherheit versteht, wurde am deutlichsten in der Nationalen Sicherheitsstrategie vom Herbst 2002 dargelegt. ${ }^{4}$ Während sich der Fokus der meisten Europäer auf die Betonung amerikanischer Macht jenseits aller Herausforderungen und die Erwägung präemptiver wie präventiver Maßnahmen gegen Bedrohungen richtete, bestand jedoch die eigentliche Stoßrichtung des Dokuments darin, eine Vision der Welt zu präsentieren, die durch freie Märkte, Menschenrechte, Rechtstaatlichkeit und Demokratie bestimmt wird und damit die Formel liefert, die Welt von den Wurzeln des Terrorismus zu befreien und die als zusätzliche Dimension um eine militärische Reaktion auf terroristische Organisationen ergänzt wird.

Auf Afghanistan als den ersten Testfall für die Bush-Administration folgte sehr rasch der Irak. Vielleicht ist es wichtig, daran zu erinnern, dass der Irak keinen neuen Problemfall darstellte, sondern einen, der bereits seit dem Ersten Golfkrieg 1991 schwelte. Zudem ist er eingebettet in den wesentlich größeren Rahmen der Suche nach Frieden zwischen Israel und den Palästinensern beziehungsweise für den Nahen und Mittleren Osten - denn die Straße zum Frieden in dieser Region verläuft durch Bagdad - sowie in Fragen des Umgangs mit den nuklearen Ambitionen Irans und der Sicherung von Stabilität in einer ölreichen Region, von der sowohl die USA als auch Europa stark abhängig sind.

Bei dem Konflikt mit Europa, der auf Grund der Entscheidung Washingtons entstand, Saddam Hussein zu entmachten, ging es weniger darum, ob man sich Saddam Husseins entledigen sollte, sondern eher darum, mit welchen Mitteln dies am besten geschehen konnte. Die europäische Vorliebe für Diplomatie, Sanktionen, Engagement und Dialog stand im Kontrast zu Bushs Methode, das Problem mit gezücktem Messer anzugehen und Möglichkeiten für Veränderungen zu eröffnen, die sowohl bessere als auch schlechtere Szenarien zur Folge haben könnten. Während es bei vielen transatlantischen Meinungsverschiedenheiten in den vergangenen Jahrzehnten mehr um die Mittel und weniger um die Ziele ging, scheint das ernsthafte Aufeinanderprallen über die Art und Weise wie mit Saddam Hussein zu verfahren sei, einen Zusammenbruch des Konsens über Prinzipien, wie man auf Bedrohungen reagiert, darzustellen. Ob dies eine Ausnahme war - es gab ja beispielsweise einen transatlantischen Konsens darüber, wie mit dem Konflikt auf dem Balkan sogar ohne UN-Mandat umgegangen werden sollte -, hängt davon ab, ob wir einen Prozess einleiten können, mit dem wir auf die zahlreichen künftigen Bedrohungen reagieren können.

\section{Handlungsfelder und Testfälle für die europäisch-amerikanische Kooperation}

Die transatlantischen Beziehungen stehen heute vor der Herausforderung zu verstehen, dass die beiderseitige Sicherheit in erster Linie durch Faktoren bedroht wird, die außerhalb des Rahmens der Beziehungen zwischen den USA und der Europäischen Union liegen. Vor allem die Region des Nahen Ostens, die am meisten Gefahren birgt, löst die stärksten Auseinandersetzungen über den Atlantik aus.

4 Vgl. The White House: The National Security Strategy of the United States of America, September 2002, http:/ /www.whitehouse.gov/nsc/nss.html (letzter Zugriff: 01.06.2005). 
Man sollte sich ins Gedächtnis rufen, dass dies keine neue Entwicklung ist. Beide Seiten des Atlantiks streiten bereits über ein halbes Jahrhundert lang, wie mit dieser unberechenbaren Region umzugehen sei. Die Konflikte zwischen Churchill und Roosevelt im Umgang mit den arabischen Ländern am Ende des Zweiten Weltkriegs, die Suez-Krise in den Fünfzigerjahren und weitere Fälle dienen als Beispiele dafür.

Parallel zu dem zunehmend auf den Nahen und Mittleren Osten gerichteten Fokus verlor Europa als geopolitisches Betätigungsfeld für die USA an Zentralität und Bedeutung. Die Konflikte von heute werden andernorts ausgetragen und nun fühlen die Vereinigten Staaten dieselbe Verwundbarkeit, die zum Beispiel in Deutschland über so viele Jahre während des Kalten Krieges spürbar war. Seit dem 11. September sehen sich die USA als das Hauptziel terroristischer Bedrohung, und eine Reaktion darauf liegt im Kern der Strategie des Weißen Hauses von George Bush. ${ }^{5}$

Die Organisationsprinzipien, mit denen wir den Kalten Krieg beschrieben haben, lassen sich nicht auf die Situation im Irak übertragen. Wir haben es nicht vorwiegend mit regulären Armeen auf beiden Seiten einer sichtbaren Linie zu tun, sondern mit einem verworrenen Netz aus Widersprüchen und Konflikten, die ständiger Änderung unterliegen. Damit wird keine andere Region der Welt die Aufmerksamkeit von Präsident Bush während der nächsten vier Jahre stärker beanspruchen als der Nahe Osten.

Im Wesentlichen sind die Hauptziele der Vereinigten Staaten in der Region über Jahrzehnte konstant geblieben: Stabilität und Sicherheit für Israel zu sichern, den Ölfluss zum Wohl der Weltwirtschaft beizubehalten und Verbindung zu den wichtigsten Akteuren in der Region wie Ägypten und Saudi-Arabien zu halten. Erst in jüngerer Zeit fügte Präsident Bush als weiteres Ziel hinzu, die Gründung eines palästinensischen Staats zu ermöglichen. Dies wird aber in vielerlei Hinsicht enorme Investitionen erfordern.

In Palästina wurden die nächsten Schritte in der Post-Arafat-Ära mit den Wahlen im Januar 2005 unternommen. Die Europäische Union ist in der Lage, die wirtschaftliche und politische Transformation zu unterstützen - und nicht nur dort, sondern in der gesamten Region. In diesem Gebiet ist Analphabetismus weit verbreitet, die Armutsquote ist hoch, das Erziehungswesen und die Medien sind nicht frei. Die wirtschaftliche Situation der Mitglieder der Arabischen Liga ist selbst schlechter als die ärmerer Mitglieder der Europäische Union. Hilfe für die fast dreihundert Millionen Menschen in der Region - deren Zahl Jahr für Jahr exponentiell steigt - ihre eigene Zukunft zu sichern, ist nicht nur wichtig für die Araber selbst, sondern von strategischem Interesse für Europa und die USA. Es ist ebenso von grundlegender Bedeutung, dass die Führer der arabischen Staaten Verantwortung für ihre eigene Entwicklung übernehmen.

Im Hinblick auf Iran stellt dessen Streben nach Nuklearwaffen einen Katalysator für Gefahren in der Region dar, sei es durch das Risiko eines Unfalls oder durch eine Eskalation der Lage. Hier sind aufeinander abgestimmte Anstrengungen mit den britischen, französischen und deutschen Vorstößen entscheidend, um die Iraner davon zu überzeugen, dass das Streben nach Nuklearwaffen die Konsequenzen nicht wert ist. Dieses Ziel mag allerdings illusorisch sein, selbst unter Annahme eines Regimewechsels in Teheran.

Während der vergangenen 25 Jahre vermieden die USA ein direktes Engagement in Bezug auf Teheran, aber nun ist die Zeit gekommen, in der die europäische Diplomatie, das Potenzial der USA sowie die Sanktionen des UN-Sicherheitsrats gefordert sind, um mit einer

5 Vgl. David Frum/Richard Perle: An End to Evil, New York, 2003 und The National Commission on Terrorist Attacks: The 9/11 Commission Report: Final Report of the National Commission on Terrorist Attacks Upon the United States, New York 2004. 
,good cop, bad cop'-Strategie eine größere Eskalation und die weitere Verbreitung von Nuklearwaffen in dieser instabilen Region zu verhindern. Die Erfahrung mit Gaddafi war vielleicht lehrreich. Die amerikanischen und europäischen Fähigkeiten zu kombinieren, eine Mischung aus Druck und Einfluss, aus Zuckerbrot und Peitsche zu finden, ist in dieser Situation entscheidend.

Und auch hier müssen wir die Tatsache berücksichtigen, dass zur nuklearen Bedrohung Sorgen um Menschenrechtsverletzungen und die Unterstützung für terroristische Gruppen, die Verzweiflung einer wachsenden Zahl junger Menschen ohne Arbeit sowie die Gefahren militanter islamischer Gruppen hinzukommen. Genauso wie wir nicht den ganzen Nahen und Mittleren Ostens vom Irak aus beurteilen dürfen, müssen wir die gesamte Region aus verschiedenen Blickwinkeln unserer gemeinsamen Sorgen betrachten.

Jegliche Diskussion über die Notwendigkeit militärischen Eingreifens in Iran analog zu den Geschehnissen im Irak ist meiner Ansicht nach unrealistisch, trotz der Kontroverse in Washington, dass solche Pläne bereits in der Schublade lägen. Man muss begreifen, dass das Scheitern der Suche nach Beweisen für die Präsenz von Massenvernichtungswaffen im Irak bei einigen Verfechtern eines militärischen Einsatzes gegen Iran das Bestreben ausgelöst hat, unzweifelhafte Beweise dafür zu sammeln, dass die Iraner ein Atomwaffenprogramm verfolgen, sofern eine unnachgiebige Haltung gegenüber Teheran von der internationalen Öffentlichkeit unterstützt wird. Zudem setzen sich viele Exil-Gruppen für die Wiedereinführung der Monarchie ein. Meine Einschätzung des aktuellen Stands der Dinge in Washington ist folgende: Die Drohung eines militärischen Einsatzes ist nicht mehr und nicht weniger als nur eine Drohung. Die Iraner könnten sich entschließen, das als Bluff zu betrachten und sogar weitere Anstrengungen unternehmen, sich Nuklearwaffen zu sichern. Dennoch ist es wichtig, die Tatsache zu verstehen, dass es in Washington sehr intensive Bemühungen gibt, eine Basis für den Wandel in der Region zu schaffen. Außenministerin Condoleezza Rice machte dies in ihrer Aussage im Januar 2005 vor dem Senat deutlich. Sie erklärte, dass sich diese Regierung ebenso für Wandel und Veränderung engagiere wie es die US-Administrationen nach dem Zweiten Weltkrieg im Ost-West-Konflikt mit der Sowjetunion tat. ${ }^{6}$

Im Hinblick auf den Irak müssen sich die USA und Europa darauf konzentrieren, wie sie die Dynamik in der nächsten Phase der Sicherung des Fortschritts bei der Entwicklung des Irak aufrecht erhalten wollen. Wer dabei welche Aufgabe übernehmen sollte, ist eine Frage, die eine erneute Diskussion über Mittel und Ziele aufwirft.

Es gibt viele andere Testfälle für die europäisch-amerikanische Kooperation: in der Ukraine, auf dem Balkan, in Afghanistan, wo es die klaren und gemeinsamen Ziele gibt, Stabilität und Sicherheit zu erhalten. Weiterhin müssen wir fortfahren zu untersuchen, wie wir mit Fragen wie der HIV-Pandemie, mit einem Zugang zu sauberem Wasser, den Millionen Menschen in Afrika nicht haben, sowie dem Problem einer angemessenen Nahrungsversorgung umgehen. Es ist ernüchternd zu bedenken, wenn uns Timothy Garton Ash sagt, dass „(a)m 11. September 2001, als die Anschläge von al-Qaida in New York und Washington mehr als dreitausend Menschenleben forderten, [...] weltweit ungefähr dreißigtausend Kinder an vermeidbaren Krankheiten [starben]. Ebenso am nächsten und am übernächsten Tag. Desgleichen an jedem einzelnen Tag des Jahres. "7

6 Vgl. die Eröffnungsrede von Dr. Condoleezza Rice vor dem Auswärtigen Ausschuss des Senats, 18. Januar 2005, http://foreign.senate.gov/testimony/2005/RiceTestimony050118.pdf (letzter Zugriff: 01.06.2005) und das vollständige Transkript der Anhörung in der New York Times, 18. Januar 2005, http://www.nytimes.com/ 2005/01/18/politics/18TEXT-RICE.html?ex=1110690000\&en=2294cf86a58edf04\&ei=5070\&oref=login\&pa gewanted=print\&position= (letzter Zugriff: 21.03 .2005$)$.

7 Timothy Garton Ash: Freie Welt. Europa, Amerika und die Chance der Krise, Bonn, 2004, S. 201. 
Wenn wir uns ansehen, wie die europäische und die amerikanische Öffentlichkeit in großem Einklang auf das tragische Desaster in Südostasien Anfang des Jahres 2005 reagierten, dann ist es bemerkenswert, wie viele Synergien und gemeinsame Ziele in einem so kurzen Zeitraum verfolgt werden konnten.

\section{Ausblick}

Die kommenden Monate werden Gelegenheiten für einen intensiven transatlantischen Dialog bieten, da Präsident Bush allein bis Juni Europa drei Besuche abgestattet haben wird. In den vergangenen drei Jahren konnte das Weiße Haus mit seiner Darlegung, wie und warum wir unsere Denkweise über Sicherheit im 21. Jahrhundert ändern müssen, nicht überzeugen. Zwar mag Bush den Status quo, den wir aus dem 20. Jahrhundert kennen, geändert haben, aber das Weiße Haus unterließ es, hinreichend zu erklären, wie und warum wir unser Denken über die Sicherheit im 21. Jahrhundert ändern sollten. Gleichzeitig kommen die Europäer mit der Erarbeitung ihres eigenen Sicherheitsdialogs und ihrer eigenen Sicherheitspolitik nur langsam voran. Auch in Zukunft werden sie mit der Gestaltung einer Europäischen Sicherheits- und Verteidigungspolitik zu kämpfen haben.

Die Ereignisse der kommenden Jahre, diejenigen, die wir voraussehen können und die, von denen wir nichts ahnen, werden zahlreiche Gelegenheiten bieten, praktische Schritte im Umgang mit den Gefahren, mit denen wir konfrontiert sind, vorzunehmen. Doch genau wie wir unser durch den Kalten Krieg geprägtes Denken überprüfen müssen, müssen wir auch die Wahrnehmung von uns selbst sowie unseren Zielen überdenken. Welche Basis bietet der ,Westen' dafür? Wo ziehen wir die Grenzen bei der Definition, wer wir sind? Wir werden vor allem eine neue Definition für das benötigen, was wir sind oder sein sollten, und weniger dafür, was wir nicht sind.

Übersetzung aus dem Englischen von Lisa Weber. 